\title{
Laser controlled tunneling in a vertical optical lattice
}

\author{
Q. Beaufils, G. Tackmann, X. Wang, B. Pelle, S. Pelisson, P. Wolf and F. Pereira dos Santo:* \\ LNE-SYRTE, UMR 8630 CNRS, Observatoire de Paris, \\ UPMC, 61 avenue de l'Observatoire, 75014 Paris, FRANCE
}

(Dated: October 14, 2018)

\begin{abstract}
Raman laser pulses are used to induce coherent tunnelling between neighbouring sites of a vertical 1D optical lattice. Such tunneling occurs when the detuning of a probe laser from the atomic transition frequency matches multiples of the Bloch frequency, allowing for a spectroscopic control of the coupling between Wannier Stark (WS) states. In particular, we prepare coherent superpositions of WS states of adjacent sites, and investigate the coherence time of these superpositions by realizing a spatial interferometer. This scheme provides a powerful tool for coherent manipulation of external degrees of freedom of cold atoms, which is a key issue for quantum information processing.
\end{abstract}

PACS numbers: 32.80.Qk, 37.10.Jk, 05.60.Gg, 37.25.+k

Trapping and manipulating cold neutral atoms in an optical lattice offers high controllability and robust quantum coherence properties, which makes it an attractive system for many applications such as quantum simulation of solid state systems [1], metrology [2, 3], and quantum information processing (QIP) [4]. One key issue in this context is the possibility to coherently control the atoms internal and external degrees of freedom. Combined with the possibility to address single sites [5], this allows for the realization of quantum logic operations [ $[$ ]

Atom transport control in an optical lattice has been previously reported using microwave fields [7], frequency, phase and amplitude modulation techniques [8 10], or an adiabatic change of the trapping potential [11, 12]. In this work, we demonstrate coherent laser induced tunneling of cold atoms between neighboring sites of an optical lattice. In contrast with most previous approaches, our technique doesn't require any modification of the trapping potential. It allows an unprecedent control of the atom's external degrees of freedom (displacing the atoms by 1 to 9 lattice periods in this work) in a system showing good coherence properties (up to $1 \mathrm{~s}$ ).

Our system consists in laser-cooled ${ }^{87} \mathrm{Rb}$ atoms in the first band of a vertical one-dimensional optical lattice. Due to earth gravity, the ground energy levels of the lattice are shifted out of resonance. For a sufficiently large lattice depth $U_{l}$, tunneling is highly reduced, leading to a ladder of localized Wannier-Stark (WS) eigenstates separated by the Bloch frequency $\nu_{B}=m_{a} g \lambda_{l} / 2 h$. Here, $m_{a}$ is the atomic mass, $g$ is the gravity acceleration, $\lambda_{l} / 2$ is the distance between two adjacent lattice sites, and $h$ is the Planck constant. The WS states $\left|W_{m}\right\rangle$ are indexed by the discrete quantum number $m$ characterizing the well containing the center of the wave function $\left\langle x \mid W_{m}\right\rangle$.

We use counterpropagating Raman beams to drive coherent transitions between the ground and excited hyperfine levels $|g\rangle=\left|5^{2} S_{1 / 2}, F=1, m_{F}=0\right\rangle$ and $|e\rangle=$ $\left|5^{2} S_{1 / 2}, F=2, m_{F}=0\right\rangle$. Such a transition implies a momentum transfer of $k_{\text {eff }}=k_{1}+k_{2} \approx 4 \pi /(780$ $\mathrm{nm})$ that couples the WS states either in the same

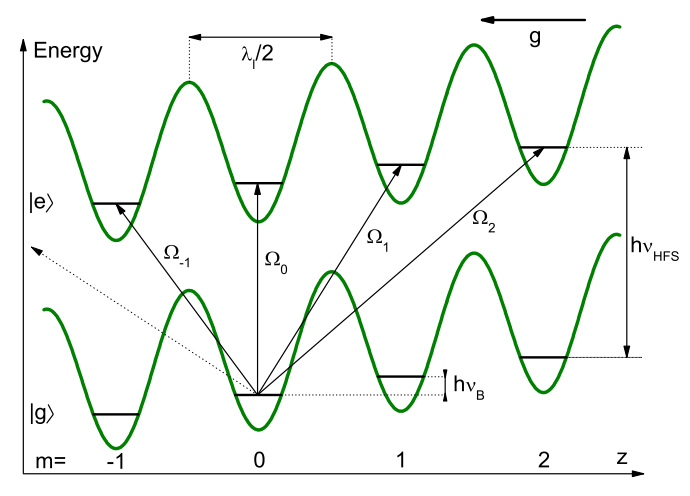

FIG. 1: Atoms in the first band of the lattice form a WannierStark ladder of eigenstates. The Raman probe laser couples the ground to the excited hyperfine level in the different WS states separated by the Bloch frenquency.

well or in neighboring wells, with a coupling strength proportional to $\left\langle W_{m}\left|e^{i k_{e f f} x}\right| W_{m \pm \Delta m}\right\rangle$. Fourier-limited widths of the resonances over excitation times larger than the Bloch period allows resolved intersite transitions $|g, m\rangle \rightarrow|e, m \pm \Delta m\rangle$, at Raman frequencies :

$$
\nu_{R}=\nu_{H F S} \pm \Delta m \times \nu_{B}
$$

where $\nu_{H F S}$ is the hyperfine splitting and $\Delta m$ is the number of lattice wells separating the two coupled WS states. The energy spectrum of our system is schematically illustrated in Fig. 1.

Coupling between neighboring wells can be efficiently tuned using the lattice depth when $k_{l}$ is close to $k_{e f f}$, where $k_{l}$ is the optical lattice wave vector [13]. We therefore use a mixed trap configuration with a blue detuned lattice generated by a single mode frequency doubled $\mathrm{Nd}: \mathrm{YVO}_{4}$ laser $\left(\lambda_{l}=532 \mathrm{~nm}\right.$, beam waist $\left.600 \mu \mathrm{m}\right)$ that provides only vertical longitudinal confinement, superposed with a red detuned $(\lambda=1064 \mathrm{~nm}$, beam waist $200 \mu \mathrm{m}) \mathrm{Yb}$ fiber laser providing transverse confinement (see Fig. 2). To load this dipole trap, we first 


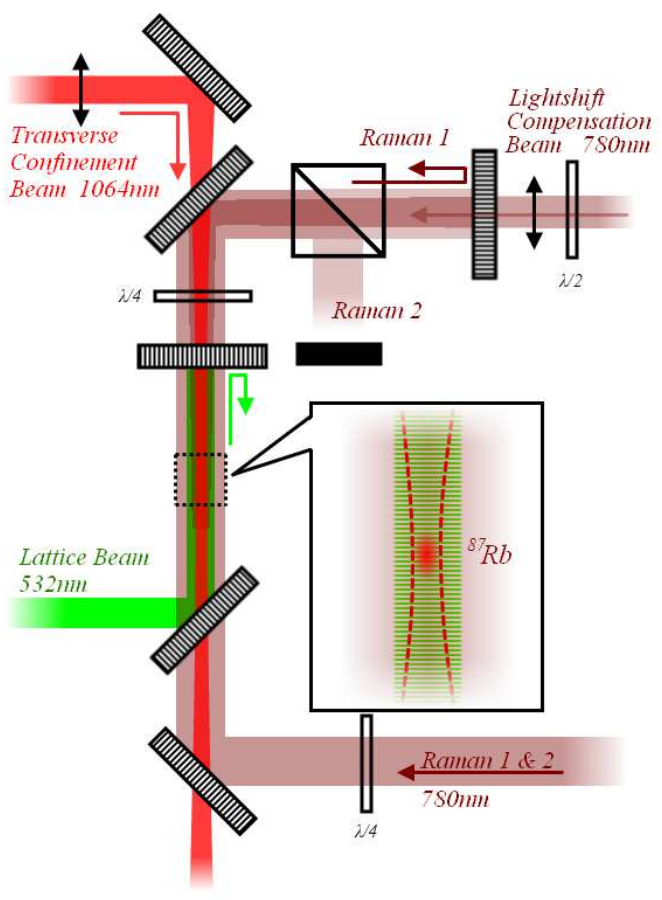

FIG. 2: Experimental setup for the optical trapping and Raman intersite transitions. The different beams are superposed using dichroic mirrors. The Raman beams are also superposed and one of them is retro-reflected to allow counterpropagating transitions.

accumulate up to $10^{7}$ atoms in a 3D-Magneto-Optical trap (MOT) fed by a 2D-MOT. The cloud is then cooled down to $2 \mu \mathrm{K}$ by a far detuned molasses, at the end of which we switch off the lasers to let the untrapped atoms fall. At our low lattice depth $\left(U_{l} \simeq 4 E_{R}\right.$ (where $E_{R}=\left(\hbar k_{l}\right)^{2} /\left(2 m_{a}\right)$ is the lattice recoil energy), only the first band has a non-negligible lifetime and is populated with about $10^{5}$ atoms vertically distributed along $10^{4}$ sites (the second band is centered at $5 E_{R}$ already above the lattice depth). The atoms accumulated in all the Zeeman sublevels of $\left|5^{2} S_{1 / 2}, F=2\right\rangle$ are depumped to $\left|5^{2} S_{1 / 2}, F=1\right\rangle$ and then optically pumped (95\% efficiency) on the $\left|5^{2} S_{1 / 2}, F=1\right\rangle \rightarrow\left|5^{2} P_{3 / 2}, F=0\right\rangle$ transition to the $\left|5^{2} S_{1 / 2}, F=1, m_{F}=0\right\rangle$ Zeeman sublevel, which is sensitive to stray magnetic fields only to second order. The remaining $5 \%$ unpolarized atoms can easily be removed from the trap with a pushing beam. Our fluorescence detection scheme, based on a time of flight measurement similar to the one used in atomic clocks and inertial sensors, allows us to measure the atomic populations in the two hyperfine states after releasing the atoms from the trap [14]. The Raman transitions are driven by two counterpropagating beams at $780 \mathrm{~nm}$ circularly polarized, detuned from the atomic transition by about 3 $\mathrm{GHz}$, and aligned along the direction of the optical trap beams. The beams are collimated with a $1 / e^{2}$ radius of

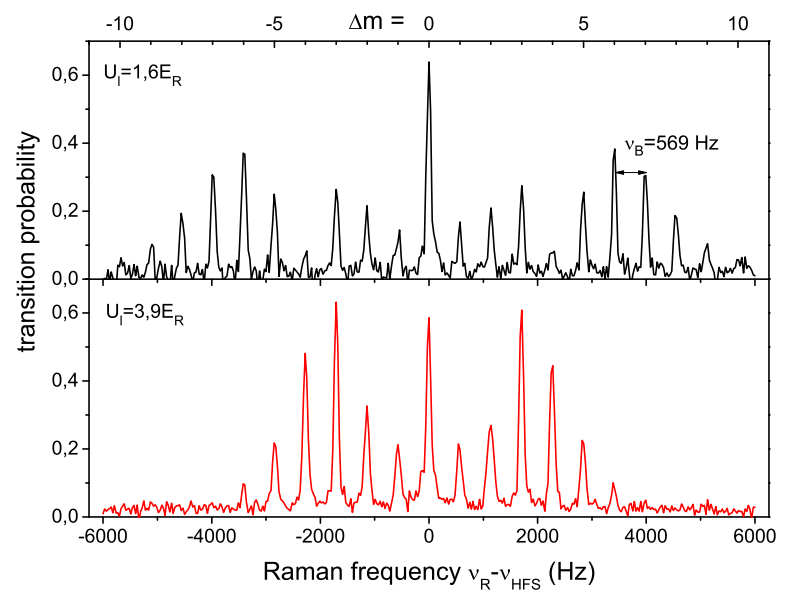

FIG. 3: Raman spectra for two different lattice depths, showing evidence of transitions between up to 9 neighboring lattice sites, each having a different Rabi frequency according to equation 1 The excitation time is $10 \mathrm{~ms}$, which is smaller than the duration of a $\pi$ pulse for each transition. The peaks are separated by the Bloch frequency of our system $\nu_{B} \approx 569$ $\mathrm{Hz}$.

$1 \mathrm{~cm}$, ensuring a good intensity homogeneity along the transverse size of the trap (about $200 \mu$ m radius).

Fig. 3 shows two typical Raman spectra of the transition probability as a function of the Raman frequency $\nu_{R}$, taken for two different lattice depths. Transitions between the two hyperfine levels at Raman frequencies equal to the hyperfine splitting plus or minus an integer number $\Delta m$ of Bloch frequencies $\left(\nu_{B} \approx 569 \mathrm{~Hz}\right.$ in our system) are the signature that the atoms actually tunneled across $\Delta m$ lattice sites. For those scans, the intensities in the Raman laser beams were 0.25 and 0.54 $\mathrm{mW} / \mathrm{cm}^{2}$. The resulting Rabi frequencies $\Omega_{\Delta m}$, different for each transition, are always smaller than the Bloch frequency, so that each peak is well resolved. The ratio between the Raman intensities was chosen to cancel the differential light shift of the hyperfine transition induced by them 15]. The Rabi frequency for each transition $\Delta m$ is written [16]:

$$
\Omega_{\Delta m}=\Omega_{U_{l}=0}\left\langle W_{m}\left|e^{-i k_{e f f} x}\right| W_{m \pm \Delta m}\right\rangle
$$

where $\Omega_{U_{l}=0}$ is the Rabi frequency in free space. Due to the translational symmetry of the WS states, $\Omega_{\Delta m}$ doesn't depend on the initial well index $m$ but only on the absolute value of $\Delta m$ [16]. It also depends on the lattice wavelength $\lambda_{l}$ and depth $U_{l}$, which is an important feature of this experiment, as it induces a spatial inhomogeneity on the Rabi frequency seen by the trapped atoms via the transverse inhomogeneity of the lattice depth in the trap. The damping induced on the Rabi oscillations by this inhomogeneity is the main limitation on the trans- 


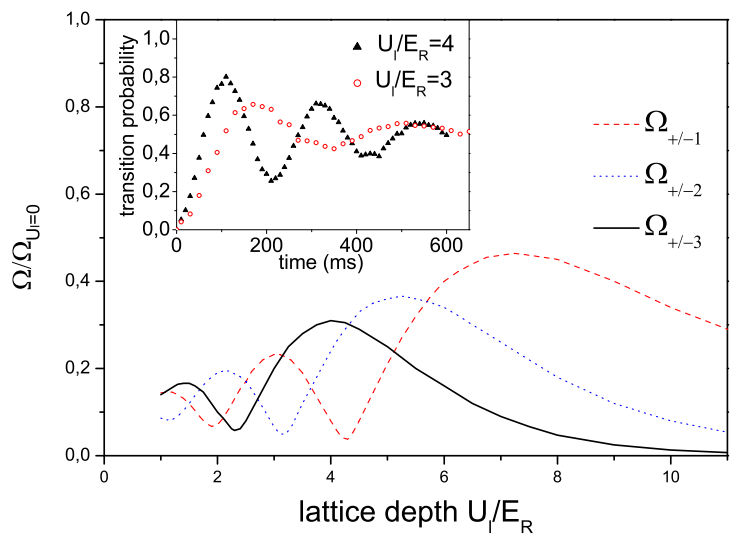

FIG. 4: Calculation of the normalized Rabi frequencies for $\Delta m= \pm 1, \Delta m= \pm 2$ and $\Delta m= \pm 3$ transitions, as a function of the lattice depth. Inset : Experimental Rabi oscillations on the transition $\Delta m=-3$, for $U_{l}=4 E_{R}$ and $U_{l}=3 E_{R}$.

fer efficiency of the Raman transitions.

We calculated $\Omega_{\Delta m}$ for the parameters of our system, as a function of $U_{l}$ and for various values of $\Delta m$. The result is shown in Fig. 4 for $\Delta m= \pm 1, \Delta m= \pm 2$ and $\Delta m= \pm 3$. To limit the lattice depth inhomogeneity due to the transverse extension of the atomic cloud, the $1 / e^{2}$ waist of the beam providing transverse confinement is smaller than the one of the lattice beam. Moreover, we can choose to tune the lattice depth at a value where, for a transition of interest, the variation of the coupling with lattice depth $\Delta \Omega\left(U_{l}\right) / \Delta U_{l}$ is small, as illustrated by the inset of Fig. 4. On this graph, we compare the shape of Rabi oscillations at resonance for the $\Delta m=-3$ transition and for two different lattice depths. Besides a difference in the period of the Rabi oscillations, we observe that the best contrast is obtained for $U_{l} \simeq 4 E_{R}$, where the coupling inhomogeneity is lower, allowing us to reach a transfer efficiency of about $80 \%$. The lattice depth is estimated by measuring the Rabi frequencies for different transitions and comparing them to the calculation.

We investigated the question of the coherence time of the trapped states. As a diagnosis tool, we performed Ramsey spectroscopy on the $|g, m\rangle \rightarrow|e, m+3\rangle$ transition. As there is no initial atomic coherence from one site to it's neighbors, the atoms distributed in many lattice sites can be treated as independent interferometers. The phase is read out by the measurement of the internal atomic state population. Our interferometer consists in two Raman $\pi / 2$ pulses of frequency $\nu_{R}$ scanned close to $\nu_{H F S}+3 \nu_{B}$, separated by a time interval $T$. The intensity of the Raman lasers was chosen for the Rabi frequency $\Omega_{\Delta m=3} / 2 \pi$ to be much smaller than $\nu_{B}$, in order to ensure a good separation with neighboring transitions. The

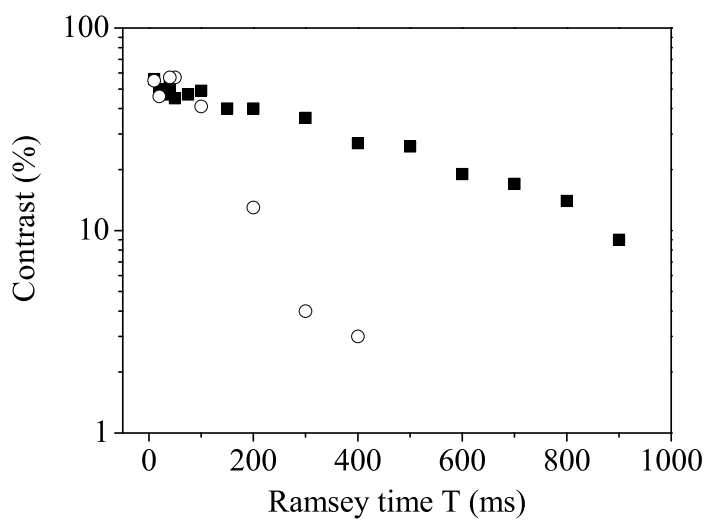

FIG. 5: Contrast of the interferometer versus Ramsey interrogation time $T$. Open circles (resp. black squares) display the contrast without (resp. with) the light shift compensation beam.

duration of the $\pi / 2$ pulses is then $\tau_{\pi / 2}=5.5 \mathrm{~ms}$. Fig. 5 shows as open circles the evolution of the contrast as we increase the interrogation time $T$. The contrast at short $T$ is about $65 \%$, which is coherent with the $80 \%$ efficiency of each of the two Raman $\pi / 2$ pulses. When increasing $T$, the contrast decreases rapidly, which is due to the transverse inhomogeneity of the differential light shift induced on the hyperfine transition by the Gaussian profile of the IR laser used for transverse trapping [17]. This position dependent frequency shift induces an inhomogeneous broadening along the transverse direction, which is the main effect limiting the coherence lifetime in the lattice trap.

Many schemes have been proposed and demonstrated to cancel this source of inhomogeneous dephasing in an optical trap [18 20]. One particularly efficient method in our case is to add a low power laser beam, mode matched with the transverse trapping beam, and whose frequency is tuned between the two hypefine levels, as reported in 21]. This beam compensates the differential ligthshift induced by the transverse trapping light with a laser power of only a few tens of $\mathrm{nW}$, so that decoherence due to photon scattering is negligible at the experiment's time scale. In practice, we use as a compensating beam a fraction of one of the two Raman beams, with an additional detuning of $80 \mathrm{MHz}$ in order to prevent undesired Raman transitions. This beam is superposed with the IR laser (see Fig. 2), and its size, position and power are adjusted to optimize the contrast of the interferometer for long interrogation times of several hundreds of milliseconds, for which otherwise the contrast is zero. In our case, for an IR power of about $2 \mathrm{~W}$, the differential light shift is compensated with a power of $12 \mathrm{nW}$. Fig. 5 displays as black squares the evolution of the contrast vs $\mathrm{T}$ with the compensating beam, and clearly shows the improvement 
of the lifetime of the coherence.

Finally, as a preliminary investigation, we evaluated the frequency sensitivity of the interferometer. This is motivated by the possibility to use this trapped atomic spatial interferometer for metrology applications such as gravimetry or short range forces measurement 22]. We locked the Raman lasers frequency difference to the center fringe of the interferometer, with a computer controlled servo integrator. Performing the measurement alternatively on the left and right transitions $(\Delta m= \pm 3)$ and calculating the difference of the measured frequencies allows us to measure $6 \nu_{B}$ while cancelling the frequency shifts of the hyperfine clock frequency, due to, for example, the quadratic zeeman effect and the differential lightshifts induced by the trapping lasers. For an interrogation time of $T=400 \mathrm{~ms}$ and a cycle time of $T_{c}=1.4 \mathrm{~s}$, the Allan standard deviation of the frequency difference decreases as $0.1 \mathrm{~Hz} . \tau^{-1 / 2}$ with $\tau$ the integration time in seconds. This corresponds to a statistical uncertainty on the measurement of the Bloch frequency of $6 \times 10^{-5}$ in relative value after $1 \mathrm{~s}$ integration.

Our Wannier-Stark interferometer shows great potential for metrology applications. As an example, we plan to perform it close to the reflecting surface of the lattice, which would allow the measurement of short range forces (Casimir-Polder, short range modifications of gravity) 13]. The statistical uncertainty in the measurement of the Casimir-Polder potential, assuming the performance demonstrated here, would reach $1 \%$ for a distance of $5 \mu \mathrm{m}$ and a measurement time of $1000 \mathrm{~s}$.

The technique for controlled and coherent transport of atoms demonstrated in this work is unique in terms of versatility. The high resolution reachable by the Raman transitions (up to $1 \mathrm{~Hz}$ ) suggests the possibility of selectively addressing one single lattice site, using for example the lightshift induced by a focused laser to lift the degeneracy between the transitions. Although demonstrated here for a thermal cloud, this technique is perfectly suitable for degenerate quantum gases. Besides, it also works with one photon transitions, as recently highlighted in [23]. All these features make this tool a potential candidate for the realization of quantum logic operations.

This research is carried on within the project iSense, which acknowledges the financial support of the Future and Emerging Technologies (FET) programme within the Seventh Framework Programme for Research of the European Commission, under FET-Open grant number: 250072. We also gratefully acknowledge support by Ville de Paris (Emergence(s) program) and IFRAF. G.T. thanks the Intercan network and the UFA-DFH for financial support.

Helpful discussions with A. Landragin, P. Lemonde,
A. Clairon, S. Bize, M-C. Angonin and R. Messina are greatfully acknowleged.

* franck.pereira@obspm.fr

[1] J. J. Garcia-Ripoll, M. A. Martin-Delgado, and J. I. Cirac, Phys. Rev. Lett. 93, 250405 (2004).

[2] M. Takamoto, F.-L. Hong, R. Higashi, and H. Katori, Nature (London) 435, 321 (2005)

[3] P. Cladé, E. de Mirandes, M. Cadoret, S. GuellatiKhélifa, C. Schwob, F. Nez, L. Julien, and F. Biraben, Phys. Rev. Lett. 96, 033001 (2006)

[4] H.J. Briegel, T. Calarco, D. Jaksch, J. I. Cirac and P. Zoller, J. Mod. Opt. 47, 415451 (2000).

[5] D. Schrader, I. Dotsenko, M. Khudaverdyan, Y. Miroshnychenko, A. Rauschenbeutel, and D. Meschede, Phys. Rev. Lett. 93, 150501 (2004)

[6] G. K. Brennen, C. M. Caves, P. S. Jessen, and I. H. Deutsch, Phys. Rev. Lett. 82, 10601063 (1999)

[7] L. Förster, M. Karski, J. Choi, A. Steffen, W. Alt, D. Meschede, and A. Widera, E. Montano, J. Hoon Lee, W. Rakreungdet, and P. S. Jessen, Phys. Rev. Lett. 103, 233001 (2009)

[8] M. Ben Dahan, E. Peik, J. Reichel, Y. Castin, and C. Salomon, Phys. Rev. Lett. 76, 4508 (1996)

[9] S. R. Wilkinson, C. F. Bharucha, K.W. Madison, Q. Niu, and M. G. Raizen, Phys. Rev. Lett. 76, 4512 (1996)

[10] V.V. Ivanov, A. Alberti, M. Schioppo, G. Ferrari, M. Artoni, M. L. Chiofalo and G. M. Tino, Phys. Rev. Lett. 100, 043602 (2008)

[11] O. Mandel, M. Greiner, A. Widera, T. Rom, T. W. Hänsch and I. Bloch, Nature (London) 425, 937 (2003)

[12] M. Anderlini, P. J. Lee, B. L. Brown, J. Sebby-Strabley, W. D. Phillips and J. V. Porto, Nature (London) 448, 452 (2007)

[13] F. Pereira dos Santos et al., Proceedings of the $7^{\text {th }}$ symposium Frequency Standards and Metrology, (World scientific printers, Singapore, 2009)

[14] J. Le Gouët, T. E. Mehlstäubler, J. Kim, S. Merlet, A. Clairon, A. Landragin, and F. Pereira Dos Santos, Appl. Phys. B 92, 133 (2008)

[15] D.S. Weiss, B.C. Young and S. Chu, Applied Physics B 59, 217-256 (1994)

[16] P. Lemonde and P. Wolf, Phys. Rev. A 72, 033409 (2005)

[17] S. Kuhr, W. Alt, D. Schrader, I. Dotsenko, Y. Miroshnychenko, A. Rauschenbeutel and D. Meschede, Phys. Rev. A 72, 023406 (2005)

[18] M. F. Andersen, A. Kaplan, and N. Davidson, Phys. Rev. Lett. 90, 023001 (2003)

[19] A. Derevianko Phys. Rev. Lett. 105, 033002 (2010)

[20] R. Chicireanu, K. D. Nelson, S. Olmschenk, N. Lundblad, A. Derevianko, and J. V. Porto, Phys. Rev. Lett. 106, 063002 (2011)

[21] A. Kaplan, M. F. Andersen, and N. Davidson, Phys. Rev. A 66, 045401 (2002)

[22] P. Wolf, P. Lemonde, A. Lambrecht, S. Bize, A. Landragin, and A. Clairon, Phys. Rev. A 75, 063608 (2007)

[23] L. Yi, S. Mejri, J. J. McFerran, Y. Le Coq, and S. Bize, Phys. Rev. Lett. 106, 073005 (2011) 